\title{
Shape Effects of Iron Nanowires on Hyperthermia Treatment
}

\author{
Wei-Syuan Lin, ${ }^{1}$ Hong-Ming Lin, ${ }^{1}$ Hsiang-Hsin Chen, ${ }^{2}$ \\ Yeu-Kuang $\mathrm{Hwu}^{2}$, and Yuh-Jing $\mathrm{Chiou}^{3}$ \\ ${ }^{1}$ Department of Materials Engineering, Tatung University, Taipei 104, Taiwan \\ ${ }^{2}$ Institute of Physics, Academia Sinica, Nankang, Taipei 115, Taiwan \\ ${ }^{3}$ Department of Chemical Engineering, Tatung University, Taipei 104, Taiwan
}

Correspondence should be addressed to Hong-Ming Lin; hmlin@ttu.edu.tw

Received 7 October 2012; Accepted 12 May 2013

Academic Editor: Haiping Hong

Copyright (C) 2013 Wei-Syuan Lin et al. This is an open access article distributed under the Creative Commons Attribution License, which permits unrestricted use, distribution, and reproduction in any medium, provided the original work is properly cited.

\begin{abstract}
This research discusses the influence of morphology of nanomagnetic materials (one-dimensional iron nanowires and zero-dimensional iron nanoparticles) on heating efficiency of the hyperthermia treatment. One-dimensional iron nanowires, synthesized by reducing method in external magnetic field, are explored in terms of their material properties, magnetic anisotropy, and cytotoxicity of EMT-6 cells. The magnetic anisotropy of an array of nanowires is examined in parallel and perpendicular magnetic fields by VSM. For the magnetic hyperthermia treatment tests, iron nanowires and nanoparticles with different concentrations are heated in alternating magnetic field to measure their actual heating efficiency and SLP heating properties. The shape effects of iron nanomaterials can be revealed from their heating properties. The cytotoxicity of nanowires with different concentrations is measured by its survival rate in EMT- 6 with the cells cultivated for 6 and 24 hours.
\end{abstract}

\section{Introduction}

According to the World Health Organization (WHO), cancers were the top causes of death all over the world. In 2008 alone, 7.6 million victims died from cancers, accounting for $13 \%$ of the total death toll [1]. Currently, cancer can be treated by chemotherapy, radiation therapy, surgery, gene therapy, and immunotherapy, and so forth. Conventional hyperthermia treatments include ultrasound, radiofrequency, microwaves, infrared radiation, and tubes with hot water. Some of them will cause side effect all over the patients' bodies during the treatment [2-4].

This research is focused on the magnetic targeted hyperthermia treatments. This treatment can directly eliminate the cancer cells without damaging the normal ones. Most importantly, the medication applied on the surfaces of magnetic materials can directly perform the treatment $[5,6]$. Magnetic targeted hyperthermia treatments are conducted by placing magnetic targeted medicines on the tumor and imposing the alternating magnetic field $(\sim \mathrm{kHz}-\sim \mathrm{MHz})$ on it $[7,8]$.

To generate heat in an alternating field, magnetic materials activate more than four different known mechanisms, which are generation of eddy currents, hysteresis losses, relaxation losses, and frictional losses, and so forth. There are two modes, rotational (Brownian) and Neel, in which the relaxation losses in single-domain MNPs occur. The magnetic moment fixed along the crystal easy axis revolves away towards the external field in the Neel mode. Similar to the hysteresis loss in multidomain magnetic particles, the Neel mechanism can also generate heat by means of "internal friction" caused by the movement of the magnetic moment in an external field. In the Brownian mode, however, the interaction between a thermal force and viscous drag in the suspending medium will influence the particle movement significantly. The whole particle moves towards the external field with the moment locked along the crystal axis instead. This phenomenon serves to account for the mechanical friction component in a given suspending medium. Applying the mechanism mentioned above, the local temperature of the tumor can rise up to $42-50^{\circ} \mathrm{C}$, with that the cancer cells will be killed [9-11].

The important factor for magnetic heating is the specific loss power (SLP) (also called the specific absorption rate (SAR)). SLP is equal to $C \times m_{\text {sample }} / m_{\text {metal }} \times \Delta T / \Delta t$, where $C$ is the specific heat capacity of sample $\left(C_{\text {water }}=4.185 \mathrm{~J} / \mathrm{g}^{\circ} \mathrm{C}\right)$, 
$m_{\text {sample }}$ is the mass of sample, $m_{\text {metal }}$ is the mass of the magnetic material in sample, and $\Delta T / \Delta t$ is the slope of the heating curve. And SLP is very sensitive to the material properties $[7,12,13]$.

The factors that affect the biodistribution of polymeric iron nanoparticles (NPs) include the size, shape, hydrophobic/hydrophilic balance of the surface, and surface charge [14]. Thus, this experiment explores the characteristic differences of heating effectiveness between $1 \mathrm{D}$ iron nanowires (NWs) and commercial iron nanoparticles (NPs) in hyperthermia treatments. Iron NWs reveal a special property that may easily destroy the cancer cells by its $1 \mathrm{D}$ spinning radius. The cellular toxic tests of the nanoiron are also conducted for the iron NWs in further studies.

\section{Experimental}

Iron (III) chloride hexahydrate $\left(\mathrm{FeCl}_{3} \cdot 6 \mathrm{H}_{2} \mathrm{O}, 98 \%\right.$, assay) and sodium borohydride ( $98 \%$, assay) are purchased from SigmaAldrich Company and Acros Organics Company, respectively. Ethanol (99.5\%, assay) was obtained from Shimakyu Pure Chemicals Company. Iron NWs are fabricated by the reduction of iron (III) chloride under high magnetic field. The aqueous iron (III) chloride hexahydrate $(0.5 \mathrm{M}, 0.2 \mathrm{~mL})$ is diluted with $5 \mathrm{~mL}$ of aqueous dextran ( $40 \mathrm{ppm})$ and the iron (III) precursor is kept in an external magnetic field of about 2000 gauss. The argon gas is bubbling through the solution to purge the dissolved oxygen. The reducing agent, $\mathrm{NaBH}_{4}$, is added dropwisely under Ar. After 5 minutes, the products are washed with ethanol for several times and dried at $95^{\circ} \mathrm{C}$ [15]. The iron nanoparticles are obtained from Yong-Zhen Technomaterial Co., Ltd. with 10-60 nm in diameter for comparison.

$\mathrm{X}$-ray powder diffraction (XRD) analysis of samples is carried out at NSRRC 1C beam line. High-resolution images of samples are examined by field emission transmission electron microscope (FE-TEM), JEOL 2100F. Magnetic hysteresis loop is measured by using vibration sample magnetization (VSM, Lakeshore) at room temperature.

The hyperthermia device (Power cube 64/900) used in this study is produced by AREZZO, President Honor Ind. Co., Ltd., Taiwan. The experimental samples are placed in the inductive coil heater of $3 \mathrm{~cm}$ diameter with an optic fiber (Luxtron One, Lambda Photometrics, UK) to measure the raise of temperature.

The biocompatibility of iron NWs is evaluated by murine breast cancer cells, EMT-6 cells. EMT-6 cells are cultured in DMEM/F12 medium with $10 \%$ of the fetal bovine serum (FBS) and $1 \%$ of penicillin-streptomycin in $5 \%$ of $\mathrm{CO}_{2}$ humidified chamber at $37^{\circ} \mathrm{C}$. After the cells are seeded on multiple plates for overnight, iron NWs dispersed in culture medium are applied to each well of culture plates. The cells are treated with different concentrations of nanoiron for 6 and 24 hours. The control cell is treated in the normal culture medium without adding iron nanowires. After incubation, the cells are directly counted by trypan blue exclusion assay for assaying cellular viability.

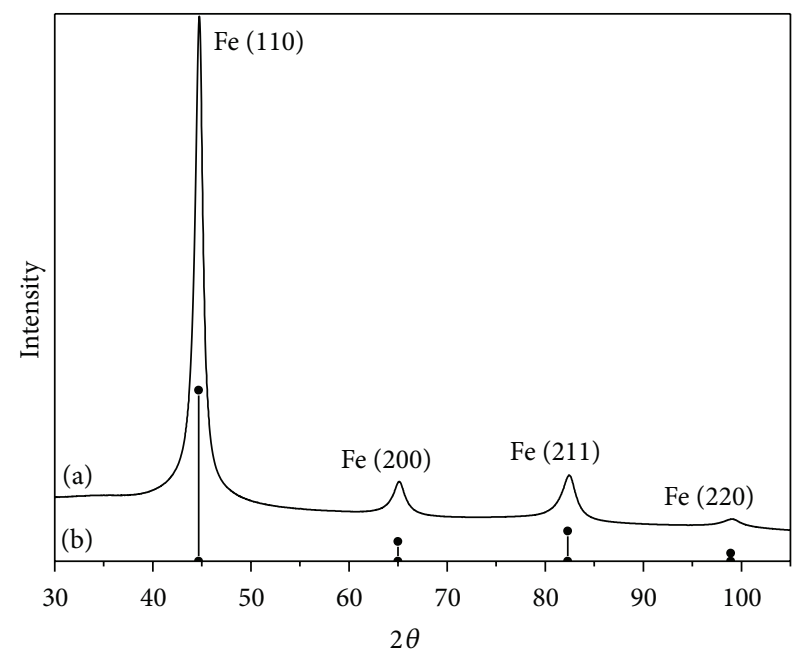

- (a) Iron NWs

-•- (b) JCPDS file 65-4899

FIGURE 1: X-ray diffraction patterns of (a) iron NWs; (b) JCPDS file 65-4899.

\section{Results and Discussion}

XRD pattern of iron NWs and JCPDS file 65-4899 are shown in Figure 1. The diffraction angles of JCPDS file 65-4899 at $2 \theta=44.662^{\circ}, 65.006^{\circ}$, and $82.311^{\circ}$ are related to (110), (200), and (211) planes of pure iron, respectively. This pattern shows clearly that a strong diffraction peak corresponds to the (110) plane. The growth orientation of iron NWs is highly dependent on applied magnetic field. According to these results, preferred crystal orientation of the iron NWs is in (110) direction. The lattice parameters of iron NWs are less than theoretical lattice constants. This may be due to lattice defects.

Figures 2(a)-2(d) show HR-TEM images of iron NWs. The diameter of iron NWs is around $60 \mathrm{~nm}$ with length of about 2-6 $\mu \mathrm{m}$ as indicated in Figure 2(a). The selective area diffraction (SAD) of circle area in Figure 2(a) is shown in Figure 2(d). It indicates that iron NWs exhibit bcc crystal structure. The diffraction planes are assigned to (110), (200), (220), (310), and (222) from inside to outside diffraction rings. Figure 2(c) indicates an oxide layer of about $4 \mathrm{~nm}$ is passivated on the surface of iron NWs. The oxide layer on iron NWs results from magnetite formation during the reduction process. TEM images show that the iron NWs are synthesized by pearl-linking of iron NPs due to external magnetic field. Figures 2(c)-2.1 to 2(c)-5.1 are the atoms resolution related to $\mathrm{cl}, \mathrm{c} 2, \mathrm{c} 3, \mathrm{c} 4$, and $\mathrm{c} 5$ positions in Figure 2(c), respectively. The d-spacing of Figures 2(c)-1.1, 2(c)-2.1, 2(c)-3.1, 2(c)-4.1, and $2(\mathrm{c})-5.1$ are $1.889 \AA, 1.879 \AA, 1.888 \AA, 2.022 \AA$, and $1.884 \AA$, respectively.

The magnetic hysteresis curve of iron NWs at room temperature is shown in Figure 3. The results indicate that the saturation magnetization and coercive force of iron NWs are $157.93 \mathrm{emu} / \mathrm{g}$ and $9.74 \mathrm{Oe}$, respectively. The saturation magnetization of iron NWs is lower than that of bulk iron (220 emu/g) due to the existence of a high surface/volume 


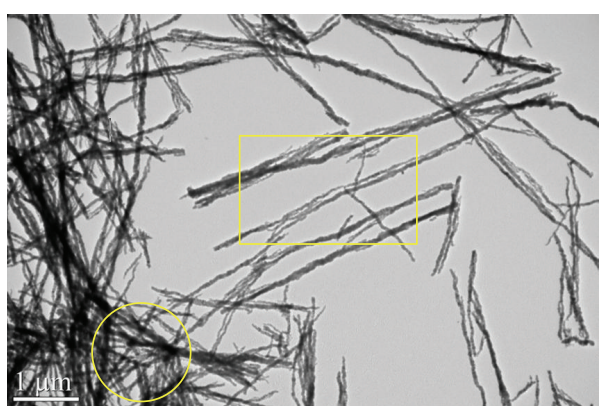

(a)

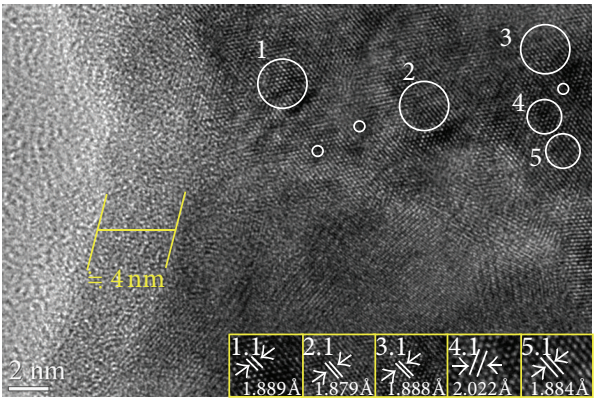

(c)

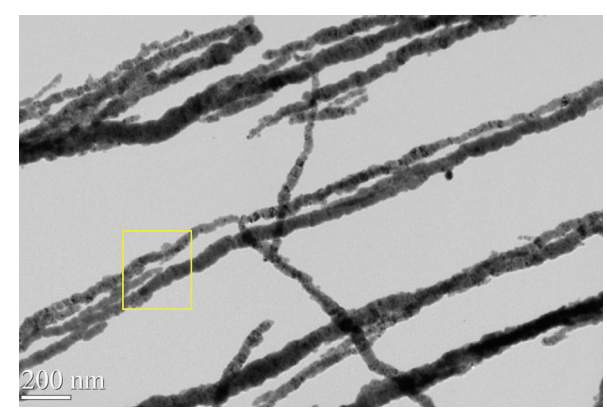

(b)

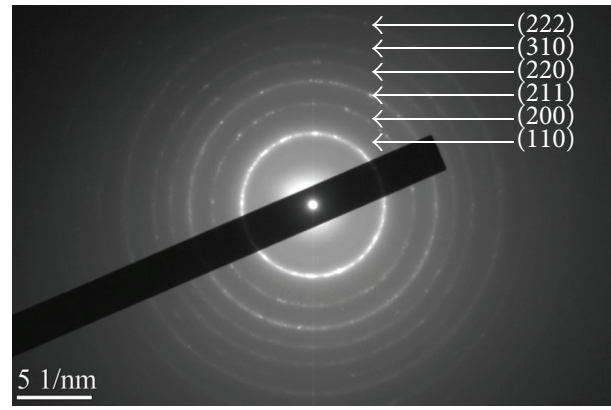

(d)

FIGURE 2: HR-TEM images of iron NWs: (a) low-magnification image; (b) high-magnification image at square area of (a); (c) HR-TEM images of iron NWs, 1.1, 2.1, 3.1, 4.1, and 5.1 are atomic resolution related to 1, 2, 3, 4, and 5 positions in (c), respectively; and (d) electron diffraction pattern at circle area of (a).

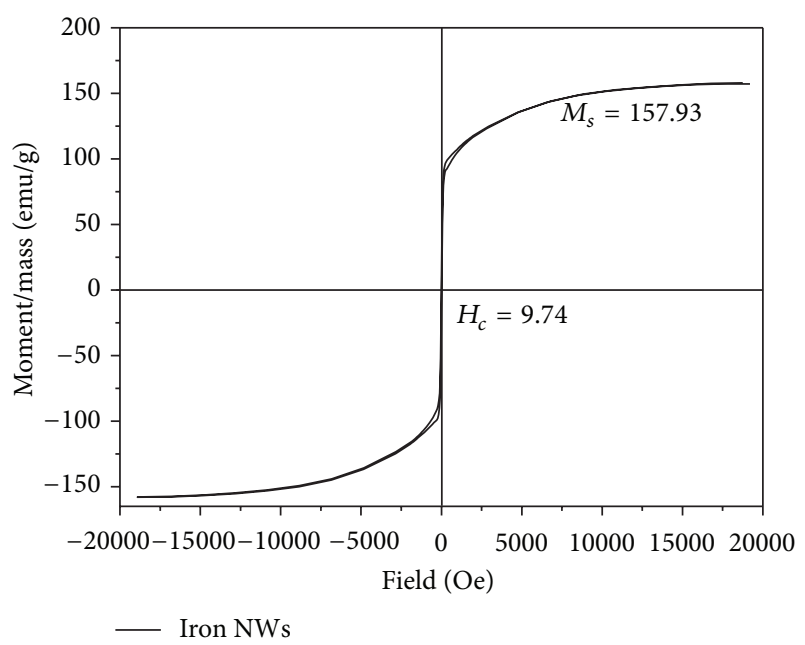

FIGURE 3: The hysteresis loops of iron NWs at $300 \mathrm{~K}$.

ratio. And structural defects may be one of the possible causes for decreasing magnetization of iron NWs.

As shown in Figure 4, the formations of an array of nanowires under (a) parallel and (b) perpendicular magnetic fields at room temperature are measured for their magnetic properties such as coercive force and squareness ratio (remanence magnetization/saturation magnetization). In Table 1 , the arrayed nanowires under the parallel magnetic field have greater squareness ratio (about $64.20 \%$ ), while those under the perpendicular magnetic field have less squareness ratio

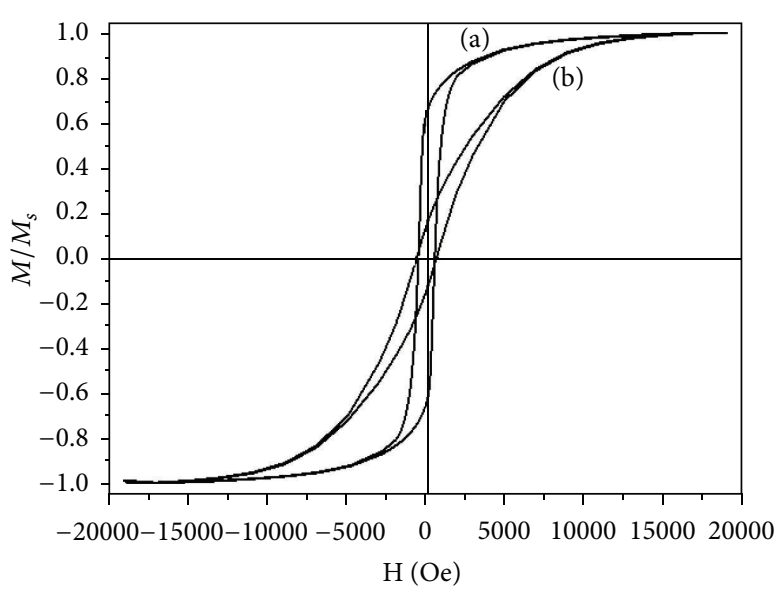

(a) Arrayed iron NWs (\|) H

(b) Arrayed iron NWs $(\perp) \mathrm{H}$

FIGURE 4: The hysteresis loops of an array of nanowires under parallel and perpendicular magnetic fields at room temperature are shown in (a) and (b), respectively.

(about 14.20\%). The former's coercive force is 100 Oe less than the latter one.

Two sets of different amounts of iron NWs $(0.1 \mathrm{mg}$, $0.25 \mathrm{mg}$, and $0.5 \mathrm{mg}$ ) and commercial iron nanoparticles $(0.5 \mathrm{mg})$ are dispersed in $1 \mathrm{~mL}$ of deionized water. SEM image of commercial iron nanoparticles produced by Yong-Zhen Technomaterial Co., Ltd. is shown in Figure 5. 
TABLE 1: The Hc (coercive force) and Mr/Ms (squareness ratio) of an array of nanowires under parallel and perpendicular magnetic fields.

\begin{tabular}{lcc}
\hline & $\mathrm{Hc}(\mathrm{Oe})$ & $\mathrm{Mr} / \mathrm{Ms}$ \\
\hline Arrayed iron NWs $(\|) \mathrm{H}$ & 520.3 & $64.20 \%$ \\
Arrayed iron NWs $(\perp) \mathrm{H}$ & 628 & $14.20 \%$ \\
\hline
\end{tabular}

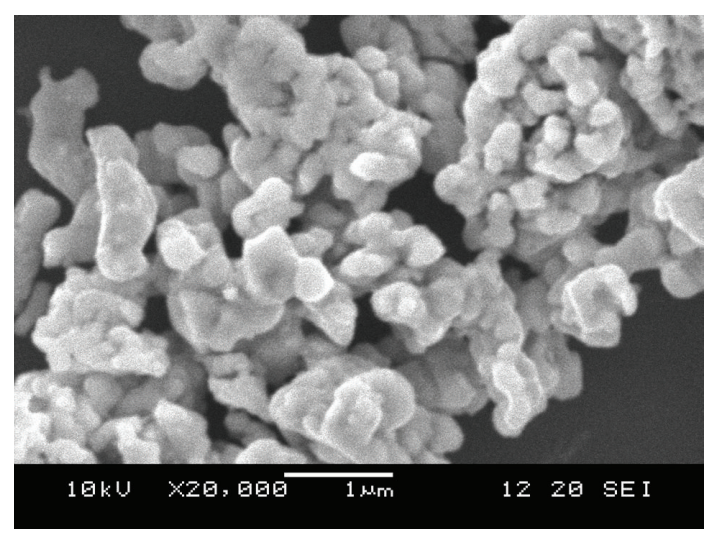

FIGURE 5: The SEM image of commercial iron nanoparticles.

Three different concentrations (100, 250, and $500 \mathrm{ppm})$ of iron NWs or commercial iron nanoparticles (500 ppm) solution in centrifuge tube is placed in the coil of inductive heater for an hour. The rising temperature of solution with time is shown in Figure 6. The maximal temperatures of iron NWs of 500,250 , and $100 \mathrm{ppm}$ can be $73.8^{\circ} \mathrm{C}, 49.6^{\circ} \mathrm{C}$, and $36.0^{\circ} \mathrm{C}$, respectively. The heating rates of commercial iron nanoparticles of $500 \mathrm{ppm}$ are $0.006^{\circ} \mathrm{C} / \mathrm{sec}$ and are listed in Table 2. When the iron nanowires and commercial iron nanoparticles have the same concentration of $500 \mathrm{ppm}$, the heating rates of iron NWs are much higher than those of the commercial iron nanoparticles. The average of SLP values for different concentrations of iron NWs and commercial iron nanoparticles are also listed in Table 2. For iron NWs, the SLP values are $619.7 \mathrm{~W} / \mathrm{g}, 535.8 \mathrm{~W} / \mathrm{g}$, and $920.8 \mathrm{~W} / \mathrm{g}$; and for commercial iron nanoparticles (500 ppm), the SLP values are $50.2 \mathrm{~W} / \mathrm{g}$. The highest SLP value $(920.8 \mathrm{~W} / \mathrm{g})$ is obtained at $100 \mathrm{ppm}$ of iron NWs. From the heating results of the iron nanowires and commercial iron nanoparticles with the same concentration of $500 \mathrm{ppm}$, the former have higher satiated temperature and efficiency than the latter one.

As mentioned above, this study presents the hypothesis in Figure 7 as an imaginary rundown. Because the iron nanowires, as compared to iron nanoparticles, have more spinning radii, the materials which are heated and stirred in the alternating magnetic field have larger reactive areas. This fact leads iron NWs to better heating efficiency than that of the iron nanoparticles.

When one-dimensional iron nanowires are applied to the tumor hyperthermia treatment, they can provide larger frictional reactive areas than zero-dimensional iron nanoparticles. This enables one-dimensional iron nanowires to have a better heating efficiency, which reduces the treatment time of the cancer patients.

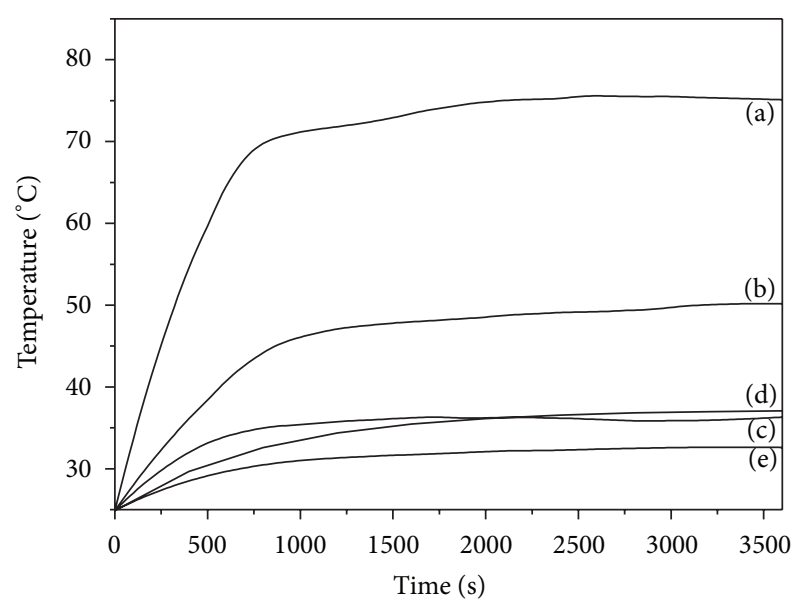

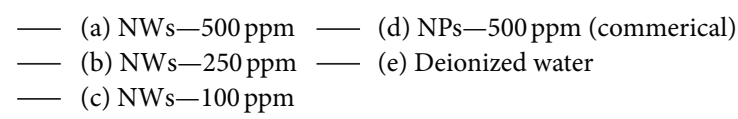

FIGURE 6: The heating effectiveness of the iron NWs in different concentrations, (a) $500 \mathrm{ppm}$ of iron NWs $(0.5 \mathrm{mg} / \mathrm{mL})$, (b) $250 \mathrm{ppm}$ of iron NWs $(0.25 \mathrm{mg} / \mathrm{mL})$, (c) 100 ppm of iron NWs $(0.1 \mathrm{mg} / \mathrm{mL})$, (d) $250 \mathrm{ppm}$ of commercial iron NPs $(0.5 \mathrm{mg} / \mathrm{mL})$, and (e) deionized water is shown for reference.
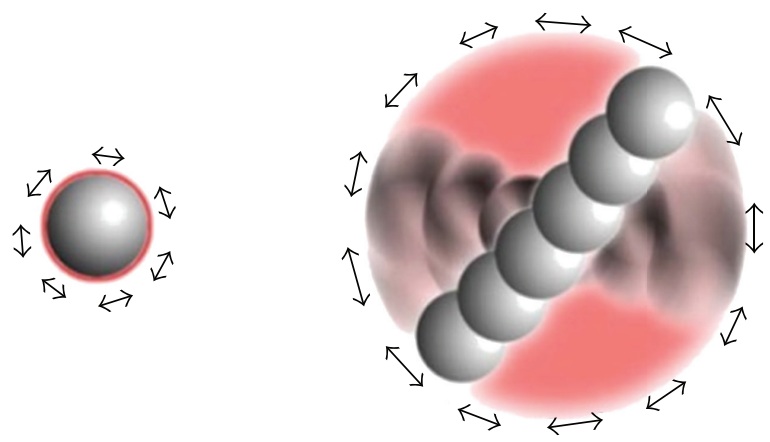

$0 \mathrm{D}$

$1 \mathrm{D}$

FIgURE 7: One-dimensional materials have large spinning radii.

TABLE 2: Heating effectiveness of iron NWs and commercial iron NPs.

\begin{tabular}{lccc}
\hline $\begin{array}{l}\text { Samples } \\
(\mathrm{ppm})\end{array}$ & $\begin{array}{c}\text { Max. Temp. } \\
\left({ }^{\circ} \mathrm{C}\right)\end{array}$ & $\begin{array}{r}\text { Heating rate } \\
\left({ }^{\circ} \mathrm{C} / \mathrm{sec}\right)\end{array}$ & $\begin{array}{c}\text { SLP } \\
(\mathrm{W} / \mathrm{g})\end{array}$ \\
\hline NWs-500 & 73.8 & 0.074 & 619.7 \\
NWs-250 & 49.6 & 0.032 & 535.8 \\
NWs-100 & 36.0 & 0.022 & 920.8 \\
NPs-500* & 37.1 & 0.006 & 50.2 \\
\hline
\end{tabular}

NPs-500: commercial iron nanoparticles.

The cytotoxicity in response to iron NWs in different concentrations $(1,10,250$, and $500 \mathrm{ppm})$ is performed by directly counting the number of live cells after incubation. Figure 8 shows the results of cytotoxicity of iron NWs in EMT-6 cells. The number of cellular vesicles appearing in the cytoplasm increases due to being exposed to iron NWs 


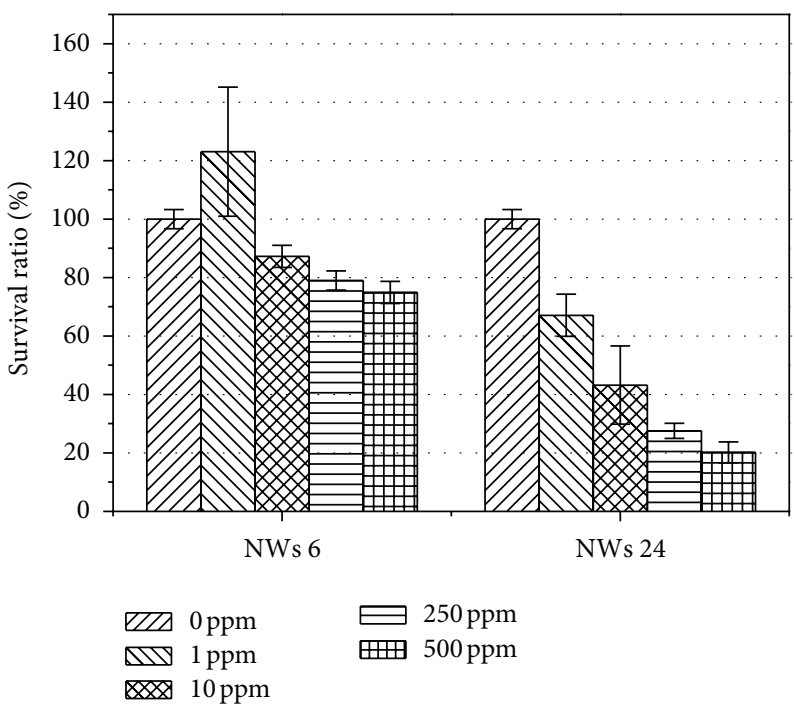

FIGURE 8: Survival ratio of iron NWs and NPs coculture with EMT-6 cells in concentrations of 0, 1, 10, 250, and 500 ppm for 6 and 24 hours.

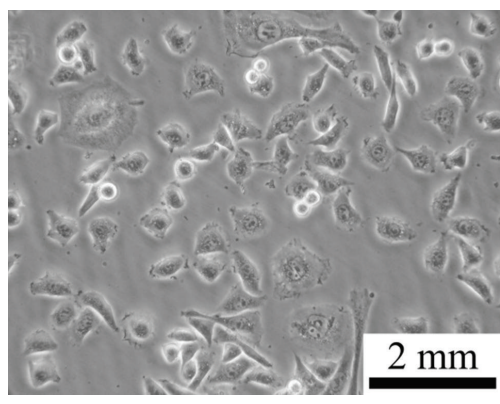

(a)

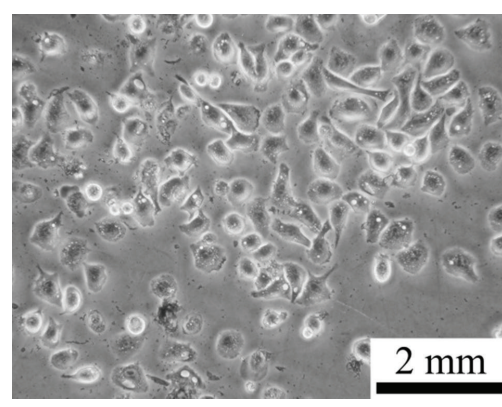

(b)

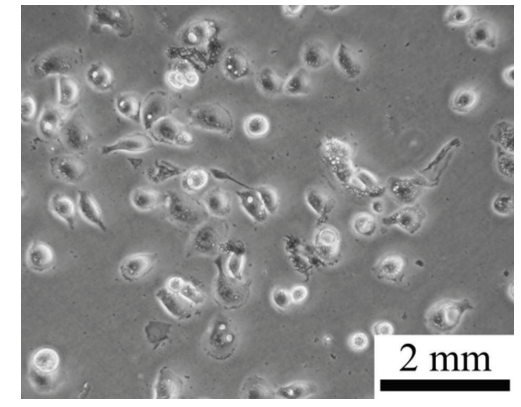

(c)

Figure 9: The optical images of (a) untreated EMT-6 cells; (b) and (c) cocultured iron NWs (10 ppm) and EMT-6 cells in 6 and 24 hours, respectively.

at lower concentrations in shorter time. After 6 hours of incubation, iron NWs shows cell survivability of more than $80 \%$. Figures 9(b) and 9(c) show the optical images of EMT-6 cocultured with iron NWs for 6 and 24 hours, respectively. The untreated cells are shown in Figure 9(a). It is also noted that the cell morphology is transformed into spherical shape.

\section{Conclusions}

This study synthesizes the iron nanowires with diameter of about $60 \mathrm{~nm}$ and length of approximately 2-6 $\mu \mathrm{m}$. Their saturation magnetization is about $157.9 \mathrm{emu} / \mathrm{g}$ while their coercive force is about $9.7 \mathrm{Oe}$. They have greater squareness ratio of about $64.2 \%$ under the parallel external magnetic field, whereas it is only about $14.20 \%$ under the perpendicular magnetic field. With regard to heating efficiency, one-dimensional iron nanowires have a better heating efficiency at the external alternating magnetic field than that of zero-dimensional ones at the same concentration of $500 \mathrm{ppm}$. The one-dimensional iron nanowires have the saturated heating temperature at about $73.8^{\circ} \mathrm{C}$ (that of the nanoparticles is only $40^{\circ} \mathrm{C}$ ) and their heating efficiency is about 0.074 (that of the nanoparticles is about 0.006 ). These results indicate the strong shape effects of nanomaterials on its heating efficiency. The cytotoxicity of the iron nanowires (10 ppm) in EMT- 6 cells undergoing 6 hours of toxic test has the mortality rate of about $80 \%$. These results indicate that with further study the iron nanowires are feasible to be used in hyperthermia therapy.

\section{Conflict of Interests}

The authors declare that they have no conflict of interests with the chemical companies (Sigma-Aldrich Company, Acros Organics Company, Shimakyu Pure Chemicals Company, and Yung-Zhen Technomaterial Co., Ltd.), instrument providers (Lakeshore, AREZZO President Honor Ind. Co., Ltd., Taiwan, Luxtron one, Lambda Photometrics, UK, NSRRC, NISP Lab in Maryland University, and Composites Lab in NTU), and research funding (NSC of Taiwan). 


\section{Acknowledgments}

The authors acknowledge the Grant from NSC of Taiwan, the support from SRRC, Taiwan, TEM/SEM from NISP Lab in Maryland University, and hyperthermia test from Bioceramics and Composites Lab in NTU, Taiwan.

\section{References}

[1] Globocan 2008, IARC, 2010, http://www.iarc.fr/.

[2] B. E. Billard, K. Hynynen, and R. B. Roemer, "Effects of physical parameters on high temperature ultrasound hyperthermia," Ultrasound in Medicine and Biology, vol. 16, no. 4, pp. 409-420, 1990.

[3] P. Wust, B. Hildebrandt, G. Sreenivasa et al., "Hyperthermia in combined treatment of cancer," The Lancet Oncology, vol. 3, no. 8, pp. 487-497, 2002.

[4] C. J. Diederich and K. Hynynen, "Ultrasound technology for hyperthermia," Ultrasound in Medicine and Biology, vol. 25, no. 6, pp. 871-887, 1999.

[5] P. Cherukuri, E. S. Glazer, and S. A. Curley, "Targeted hyperthermia using metal nanoparticles," Advanced Drug Delivery Reviews, vol. 62, no. 3, pp. 339-345, 2010.

[6] D. L. Zhao, X. X. Wang, X. W. Zeng, Q. S. Xia, and J. T. Tang, "Preparation and inductive heating property of $\mathrm{Fe}_{3} \mathrm{O}_{4}$-chitosan composite nanoparticles in an AC magnetic field for localized hyperthermia," Journal of Alloys and Compounds, vol. 477, no. 1-2, pp. 739-743, 2009.

[7] C. S. S. R. Kumar and F. Mohammad, "Magnetic nanomaterials for hyperthermia-based therapy and controlled drug delivery," Advanced Drug Delivery Reviews, vol. 63, no. 9, pp. 789-808, 2011.

[8] G. Zhang, Y. Liao, and I. Baker, "Surface engineering of core/ shell iron/iron oxide nanoparticles from microemulsions for hyperthermia," Materials Science and Engineering C, vol. 30, no. 1, pp. 92-97, 2010.

[9] R. Kötitz, W. Weitschies, L. Trahms, and W. Semmler, "Investigation of Brownian and Néel relaxation in magnetic fluids," Journal of Magnetism and Magnetic Materials, vol. 201, no. 1-3, pp. 102-104, 1999.

[10] G. Nedelcu, "Magnetic nanoparticles impact on tumoral cells in the treatment by magnetic fluid hyperthermia," Digest Journal of Nanomaterials and Biostructures, vol. 3, no. 3, pp. 103-107, 2008.

[11] G. Nedelcu, "The heating study of two types of colloids with magnetite nanoparticles for tumours therapy," Digest Journal of Nanomaterials and Biostructures, vol. 3, no. 2, pp. 99-102, 2008.

[12] R. Hergt, S. Dutz, R. Müller, and M. Zeisberger, "Magnetic particle hyperthermia: nanoparticle magnetism and materials development for cancer therapy," Journal of Physics Condensed Matter, vol. 18, no. 38, pp. S2919-S2934, 2006.

[13] F. Mohammad, G. Balaji, A. Weber, R. M. Uppu, and C. S. S. R. Kumar, "Influence of gold nanoshell on hyperthermia of superparamagnetic iron oxide nanoparticles," Journal of Physical Chemistry C, vol. 114, no. 45, pp. 19194-19201, 2010.

[14] F. Sonvico, S. Mornet, S. Vasseur et al., "Folate-conjugated iron oxide nanoparticles for solid tumor targeting as potential specific magnetic hyperthermia mediators: synthesis, physicochemical characterization, and in vitro experiments," Bioconjugate Chemistry, vol. 16, no. 5, pp. 1181-1188, 2005.

[15] W. S. Lin, Z. J. Jian, H. M. Lin et al., "Synthesis and characterization of iron nanowires," Journal of the Chinese Chemical Society, vol. 60, pp. 85-91, 2013. 

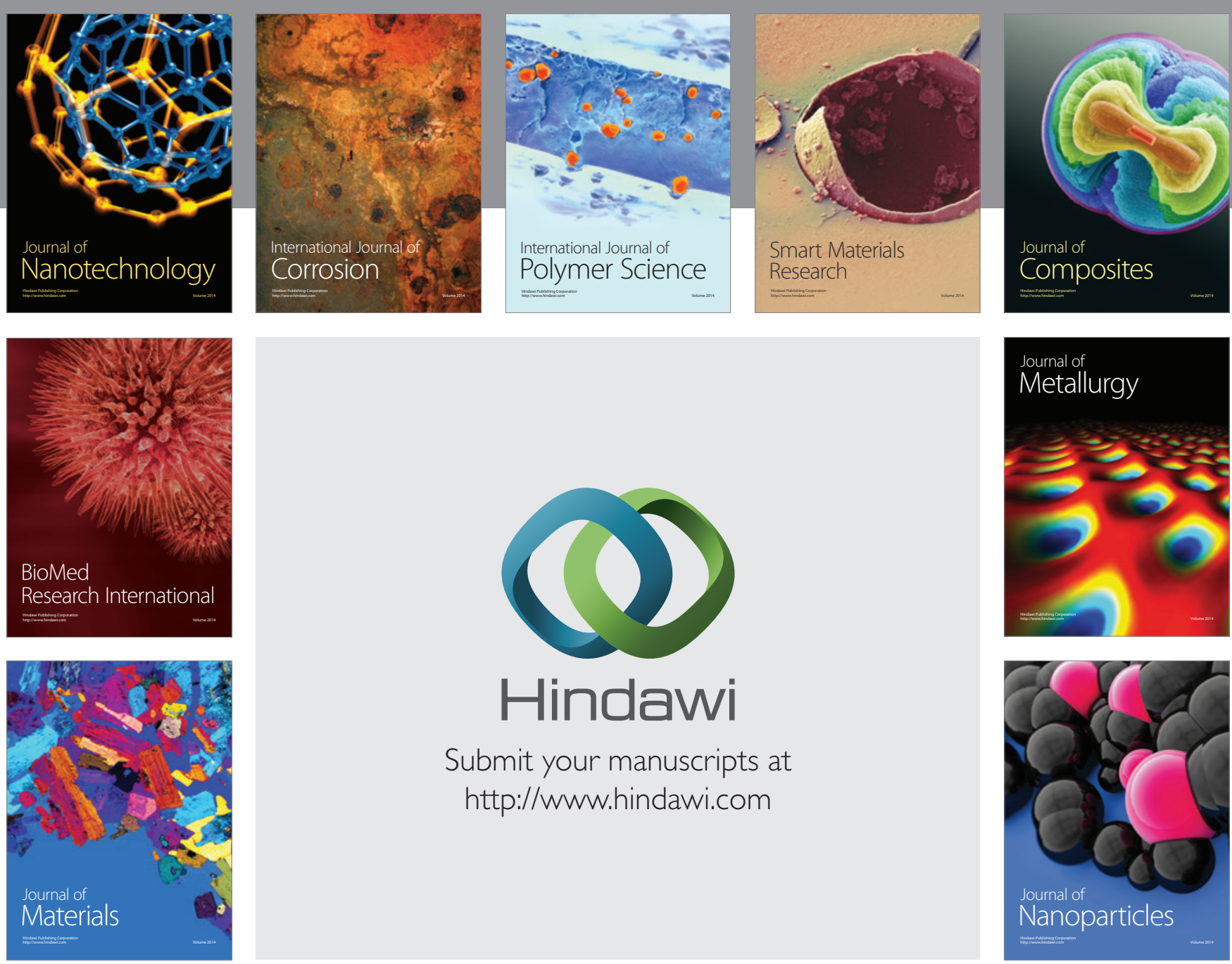

Submit your manuscripts at http://www.hindawi.com
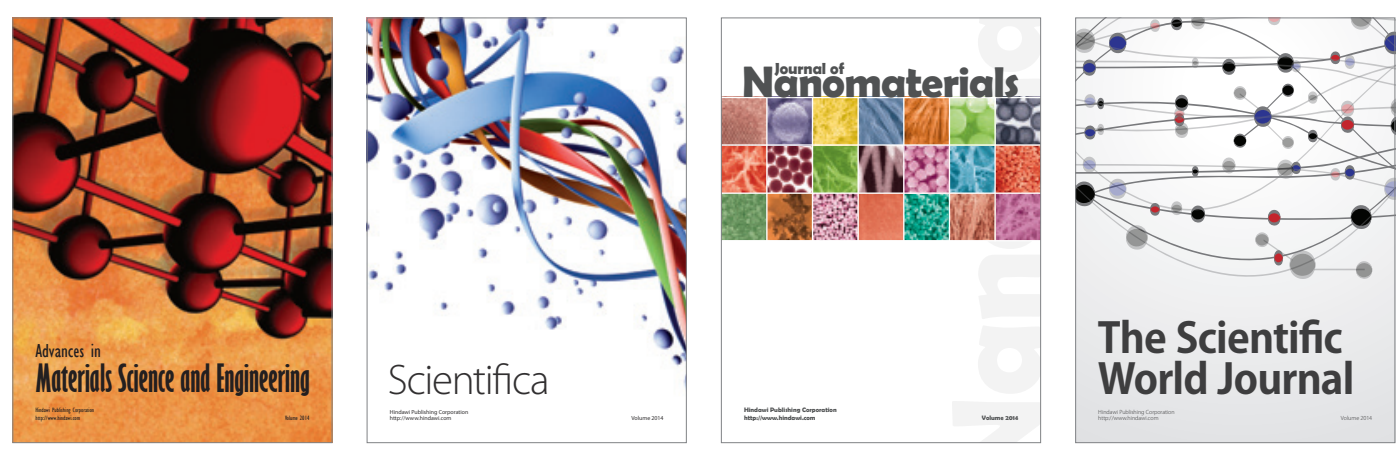

\section{The Scientific World Journal}
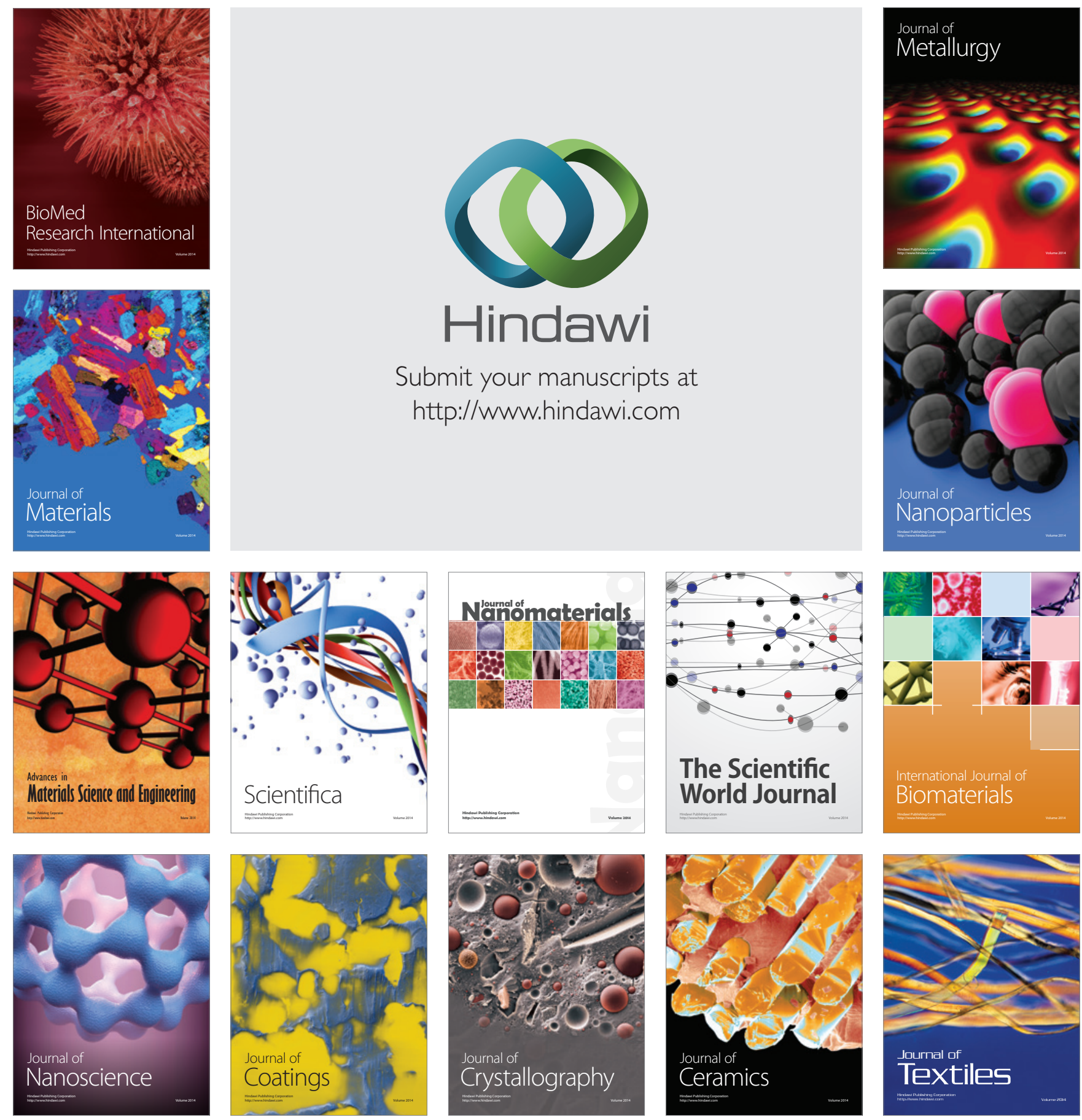